\title{
Streamlining experiment data manipulation with psychology experiment data interchange format (PXDIF)
}

\author{
GRAHAM WIDEMAN \\ San Diego State University, San Diego, California
}

\begin{abstract}
The use of small computers to automate psychology experiments has raised a number of development and logistical problems that interrupt the smooth flow of work from hypotheses to conclusions and publication. Currently, the outstanding bottleneck is the tedious and error-prone manipulation of raw experiment data in preparation for use in commercial data visualization and statistics packages. This problem is addressed by a self-describing data format PXDIF. Companion software can perform most needed manipulations automatically or with minimal experimenter involvement.
\end{abstract}

The complete experiment process is summarized in Figure 1, where three areas of concern are emphasized. Already, better and faster experiment-program development is being achieved as a result of improved development tools and higher standards in the few commercial packages. At the other end of the process, data visualization and analysis are well supported by commercial plot and stat packages, which (especially on the Apple Macintosh) are designed to produce tables and graphs that can be pasted directly into reports and publications.

What remains as a tedious and error-fraught process is the massaging of the experimental data as they are prepared for the commercial visualization and analysis packages. This situation is a burden for the experimenter if a great deal of piecemeal manipulation is necessary, and a software development overhead cost if programmers attempt to fix it.

\section{Why Data Preparation is a Problem}

There are a number of reasons why an experiment program cannot simply produce data ready for analysis programs. Part of the problem is that some intermediate processing may be required, such as the checking and removal of bad data, the joining of data gathered separately, and possibly data transformations. Other reasons are simply related to format. Most experiment programs record much more information than any one statistical format package would allow. This information may include diagnostics, experimenter comments, and auxiliary information such as date and time of day. Another reason for avoiding a pure statistical format is that it is usually desirable to feed data to at least two different packages (say,

Correspondence should be addressed to Graham Wideman, Psychology Department, San Diego State University, San Diego, CA 92182. (E-mail: graham@sunstroke.sdsu.edu) for plotting and statistical analysis), which often requires two different data arrangements.

Consequently, many programmers have chosen simply to write the data in some kind of orderly columnar format that happens to be convenient. The resulting data can be rearranged "manually" using an editor or somewhat automatically using such tools as the Unix awk program, or the programmer can write a custom application to read and rearrange the data for a particular format. All of these solutions are tedious, expensive, and error-prone.

The most distressing aspect of these solutions is that whatever or whoever is doing the rearrangement and other processing functions must have an independent knowledge of the exact meaning of each number in order to handle it properly. Given the rate at which software is changed and the sheer quantity of numbers that computercontrolled experiments are inclined to generate, this is asking for disaster.

\section{PXDIF: A Self-Describing Data Format}

The solution to this problem is a data format which incorporates a description of the data. This is what we have designed in PXDIF (psychology experiment data interchange format). Data files in this format can be printed out directly, but they are generally manipulated by a PXDIF application, such as our PXDATA. Since a PXDIF application knows how the data relate to independent variables, trials, and subjects, it can offer the user a selection of reasonable manipulations and prevent accidental manipulations that can destroy the meaning of the data.

\section{What PXDIF Records}

PXDIF's model of data is pictured in Figure 2. This is not the actual data format, rather it is simply a sketch of some of the information that is recorded. At the heart of the model is the array of data itself. This array is associated with two indexes for row and column. Rows are 
(Revision and modification path)

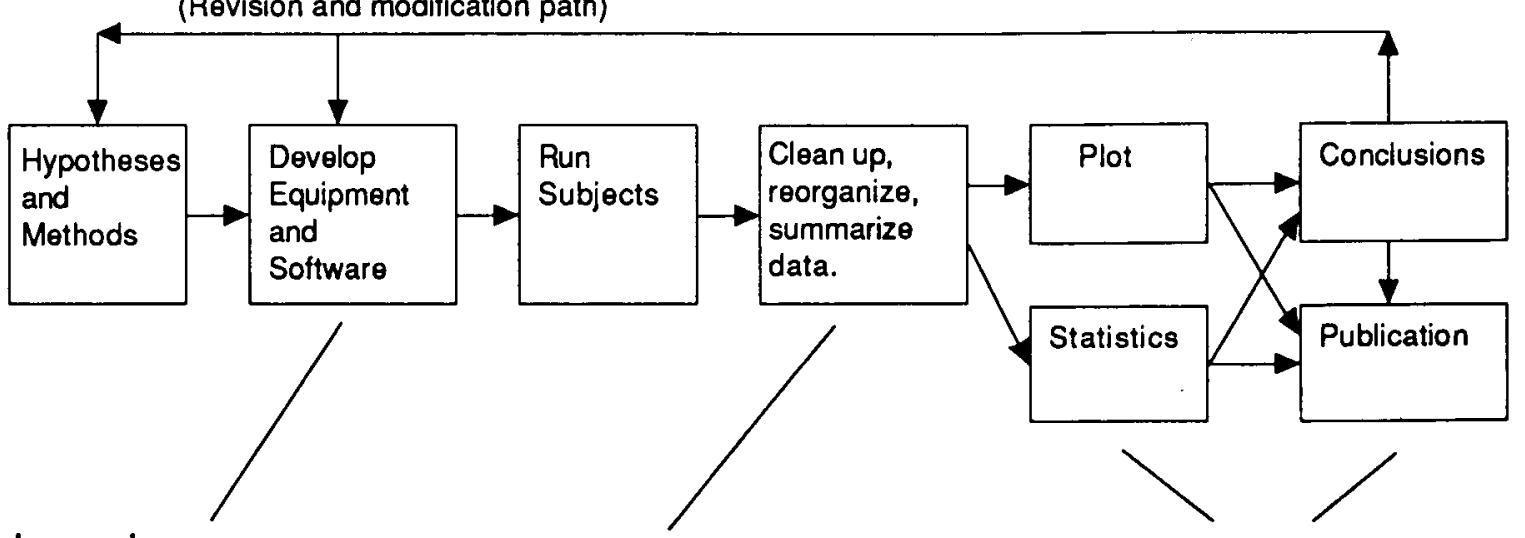

\section{Improving:}

Automated development tools, libraries, some commercial sottware help with economical software development.
The Bottleneck:

Data recorded in a form convenient for the source experiment software must be massaged before feeding to plot, stat or other packages.
Pretty Good:

Commercial plot and stat packages are readily available, and provide graphs and numbers that feod smoothly into the writing phase.

Figure 1. An outline of the experiment process, showing three areas of concern, with emphasis on the data-manipulation bottleneck.

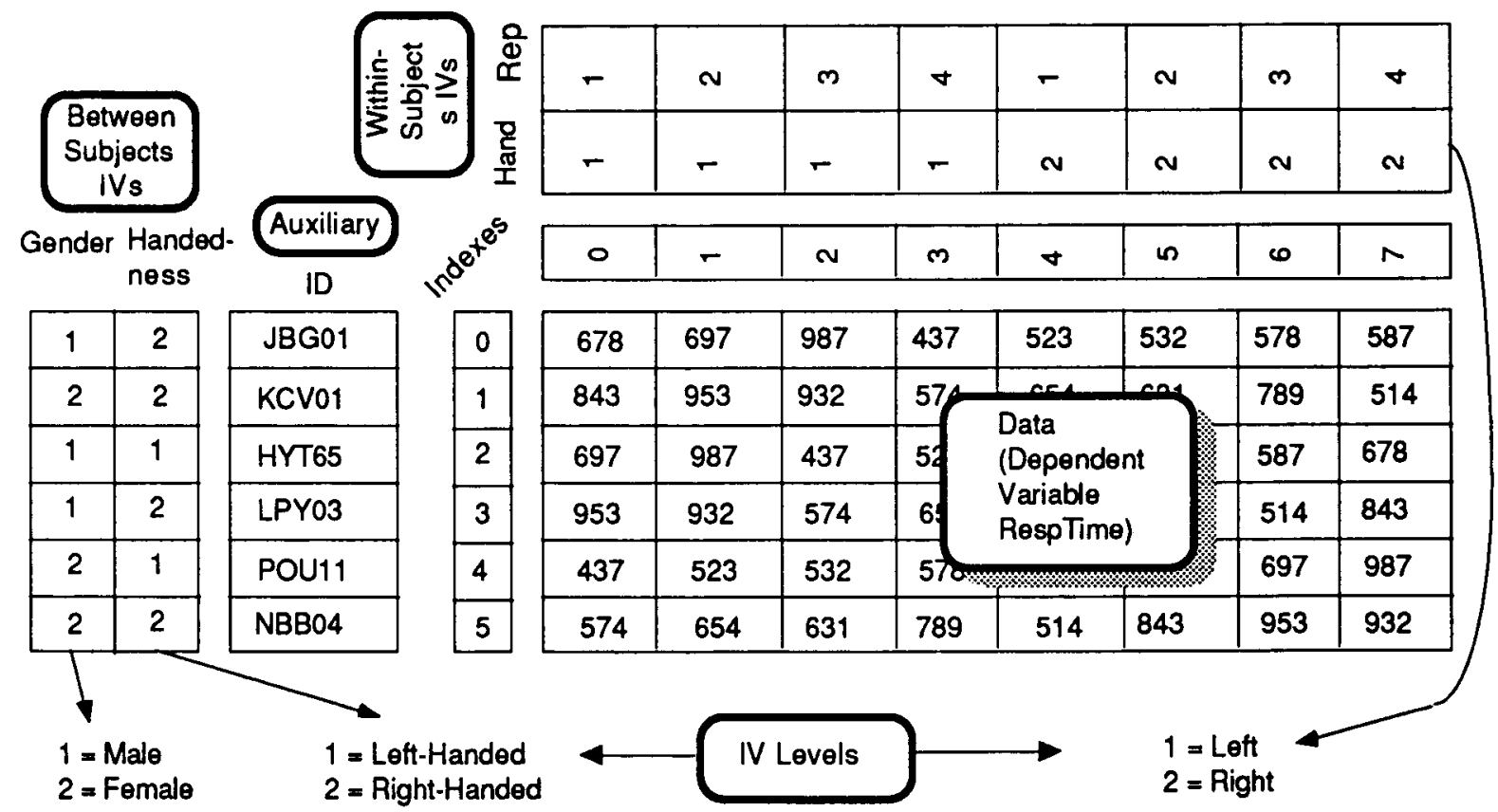

Figure 2. This sketch shows the most important PXDIF elements that relate the data to independent variables and other identifying information. 
customarily used one per subject, or one per group if the data have been reduced. Multiple columns usually correspond to repeated measures. (If there are several dependent variables, then there will be several data arrays.)

Each index is associated with independent variables (between and within subjects) and with "auxiliary" variables, which are generally for reference only, except for subject identification. Each independent variable is in turn detailed in a list of information giving meanings for each level of the independent variables.

The PXDIF file also contains some information to keep track of a file's history. This includes information about the originating experiment and any operations that have been performed on the file since then.

\section{Benefits of Self-Describing Data Files}

There are a number of things that can be done when data files describe themselves.

Output format. A PXDIF application can use the descriptive information to produce output suited to virtually any statistics, plotting, spreadsheet, or database package. Where the experimenter must make choices (e.g., which between-subject variables to include, naming of columns, etc.), the PXDIF application can offer useful alternatives or checklists.

For example, PXDATA can provide data output in a number of different stat, plot, and diagnostic forms suitable for Macintosh, PC, or mainframe statistics, plotting, or spreadsheet packages. PXDATA will provide whatever amount of labeling is supported in the destination package; for command-oriented stat packages, PXDATA supplies the commands that describe the data columns. PXDATA can also output in PXDIF, so that further manipulations can be carried out at a later time.

Summary operations. Frequently, experimental data are in a more detailed form than needed and must be summarized. For example, an experiment may collect data in small time increments, but the experimenter may wish to see hourly or daily totals or means. Or possibly the experimenter may wish to see subject-group means and feed them to a particular plotting package. This can be quickly arranged by a PXDIF application that can perform summing or meaning operations grouped by within- or between-subject independent-variable levels.

Sophisticated join operations. Data often come from different sources and must be joined into a single database. If the added data are simply from identical runs with new subjects, this is not normally a complicated process. However, real experiments are rarely so convenient. Different pieces of data for each subject may be collected in different files at different times. Trials are often run in different orders for different subjects. An experiment often evolves through several refinements, and early data are coded differently than are later data. A PXDIF application can accomplish these types of join; where experimenter intervention is required to resolve conflicts or ambiguities, the PXDIF application can offer intelligent alternatives.

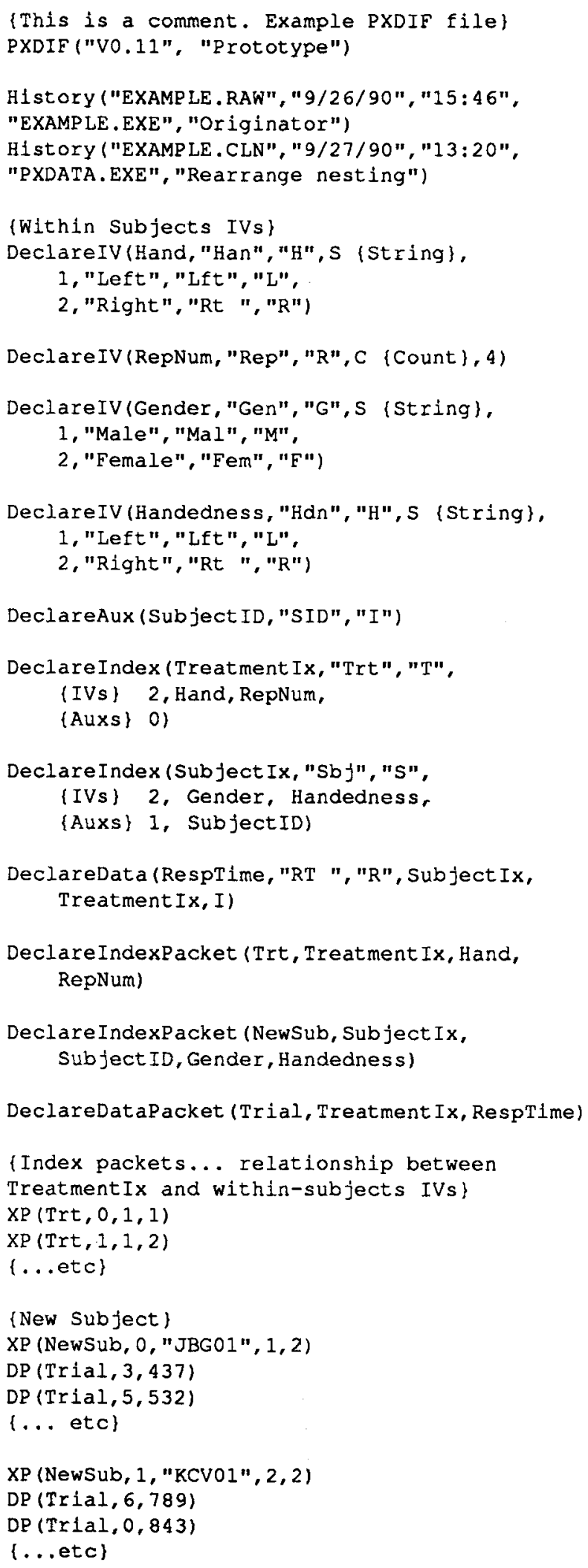

Figure 3. An example PXDF data file. (Some line parts have been abbreviated, indicated with an "...etc.") 


\section{PXDIF Format In Detail}

A sample PXDIF data file is shown in Figure 3, as it might have been generated by a session with a discretetrial experiment program. The format is simply an arrangement of text and is quite easy for an experiment programmer to program (or for an automated experimentprogram generator to produce).

In this example, the hierarchical description can be seen: description of independent variables (name and type) and all levels that are to be used; description of auxiliary (informational) variables; description of indexes, and the independent and auxiliary variables they are linked to; description of dependent variables, and the indexes that are to be used with them; description of the "index packets" that will be used for recording the relationship between independent-variable levels and indexes; description of "data packets" that will be used to record the actual data; index packets; and data packets.

The example shows how the data description is incorporated into the data file. It also demonstrates the freedom that PXDIF can offer to the experiment programmer. In this particular example, data are collected in discrete trials, within discrete subjects. However, in many experiments (particularly in animal monitoring), data for several subjects may be nested within trials or period. The programmer can be accommodated simply by producing different data-packet descriptions.

\section{What PXDIF Is Not Good For}

PXDIF is intended primarily for data that are describable fairly directly in terms of independent variables. It is not very applicable to the recording of continuous transducer data, such as EKG or sound input waveforms, whose multiple points are at a much more minute scale of detail. However, raw transducer data are often processed (e.g., to pick out pulse rate), with the goal of producing values for dependent variables relating to particular levels of independent variables. This more refined data may be conveniently handled with PXDIF.

\section{Summary}

PXDIF is a specification for a data format that can be used by experiment-control programs for saving data and by programs that manipulate, summarize, clean, and analyze those data. The features of PXDIF promote rapid development of experiment software and, most importantly, help to ensure that manipulated data are delivered intact to the postexperiment programs without requiring extraordinary skill or vigilance on the part of the user. Early implementations of PXDIF have proved very worthwhile. Our present goal is to establish a distributable standard and encourage its use by making available the PXDATA program. We welcome suggestions as we nurture PXDIF and PXDATA through increasingly useful revisions.

\section{Availability}

The full PXDIF standard and the PXDATA program are available from San Diego State University. At the time of this writing, development effort is focusing on the PC version of PXDATA; however, a Mac version is also planned. The PXDIF format itself is not system dependent. Copies of the software may be obtained by E-mail. Contact the author at graham@sunstroke.sdsu.edu for further information. 